\title{
Evaluation of a decision aid for families considering p53 genetic counseling and testing
}

\author{
Susan K. Peterson, PhD, MPH' ${ }^{1}$ Rebecca D. Pentz, PhD ${ }^{2}$, Amie M. Blanco, MS, CGC 3 , Patricia A. Ward, MS, CGC ,
} Beatty G. Watts, MS ${ }^{1}$, Salma K. Marani, MS ${ }^{1}$, Leslie Colvin James, MS, CGC ${ }^{1}$, and Louise C. Strong, $M D^{1}$

\begin{abstract}
Purpose: Li-Fraumeni syndrome (LFS) is associated with $p 53$ germline mutations, and carriers are at increased risk for multiple primary cancers. We evaluated outcomes following the administration of a video-based decision aid (DA) prior to clinical p53 genetic counseling and testing among persons who had previously participated in cancer genetics research. Methods: Fifty-seven individuals at risk for a known p53 mutation completed baseline and post-DA measures of psychological outcomes, plus knowledge and attitudes regarding p53 genetic testing. Counseling and testing uptake also was recorded. Results: At baseline, multivariate analysis showed that greater testing intention was associated with lower decisional conflict $(P<0.01)$. Compared with baseline data, multivariate analyses of post-DA outcomes showed that knowledge about LFS and genetic testing increased and decisional conflict related to testing decreased $(P<0.001)$. Mean cancer worries scores decreased among all participants $(P<0.001)$, and mean depression scores decreased for males $(P<0.05)$. Thirty-nine $(68 \%)$ completed pre-test genetic counseling and $23(40 \%)$ subsequently gave a blood sample for clinical genetic testing. Conclusion: This intervention was useful as an initial outreach and educational method for families considering p53 genetic testing, and may improve knowledge about LFS as well as psychological outcomes. Genet Med 2006: 8(4):226-233.
\end{abstract}

Key Words: p53, decision aid, genetic counseling, genetic testing, psychosocial

Li-Fraumeni syndrome (LFS) is an autosomal dominant condition that confers an increased risk for multiple primary tumors. Over $50 \%$ of families who are clinically diagnosed with LFS carry deleterious germline mutations in the $p 53$ gene. $^{1-3}$ Genetic counseling and testing of individuals from LFS families are complex because of the broad range of malignancies that comprise the spectrum of LFS (e.g., soft-tissue sarcomas, osteosarcoma, breast cancer, brain tumors, lung cancer, leukemia, melanoma, adrenocortical tumors) and because the clinical presentation varies both within and between families. ${ }^{4}$ It are estimated that $p 53$ mutation carriers have up to a $90 \%$ lifetime risk of developing a LFS-related cancer. LFS-associated cancer risks for children and young adults, especially younger women, are considerable. Men have an estimated $41 \%$ risk of developing cancer by age 45 years and a $70-90 \%$ lifetime cancer risk. In contrast, women have an estimated $84 \%$ risk of developing cancer by the age of 45 (primarily young onset breast cancer), with nearly a 100\% lifetime risk. ${ }^{4,5}$ About $20 \%$

\footnotetext{
From ${ }^{1}$ The University of Texas M. D. Anderson Cancer Center, Houston, TX; ${ }^{2}$ Winship Cancer Institute, Emory University, Atlanta, GA; ${ }^{3}$ University of California San Francisco Comprehensive Cancer Center, San Francisco, CA; ${ }^{4}$ Baylor College of Medicine, Houston, TX.

Susan K. Peterson, PhD, MPH, Department of Behavioral Science-Unit 1330, The University of Texas, M. D. Anderson Cancer Center, PO Box 301439, Houston, TX 77230-1439.

Submitted for publication October 5, 2005.

Accepted for publication January 10, 2006.

DOI: 10.1097/01.gim.0000214632.52958.01
}

of LFS-related cancers occur before the age of 20 years in $p 53$ mutation carriers of both genders. ${ }^{4}$

The significant cancer burden in LFS, coupled with the increased risk of malignancies beginning as early as young childhood, may present unique psychosocial challenges for affected families. There are no universally agreed-upon surveillance recommendations for most LFS-associated malignancies, other than earlier breast cancer screening for women ${ }^{6,7}$; thus, $p 53$ mutation carriers may receive little reassurance in terms of available strategies for the early detection of cancer. The psychosocial aspects of genetic counseling and testing for LFS have not been extensively studied. ${ }^{8,9}$ An early study compared participation in genetic testing programs for $p 53$ and $B R C A 1 \mathrm{mu}-$ tations, and found that the preliminary uptake of $p 53$ testing was significantly lower compared with BRCA1 testing uptake. ${ }^{8}$ Educational needs for $p 53$ families were suggested by participants' reasons for declining testing in this study, which included worries about upsetting themselves or their relatives with positive results, difficulty traveling to the testing center, and a lack of awareness of the hereditary nature of cancer in their families or the medical benefits of testing. ${ }^{8}$

To our knowledge, there are few informational and supportive resources to assist LFS-affected families in understanding and coping with this condition, as well as in making risk management decisions. Many LFS families, especially those outside of major urban areas, may not have access to knowledgeable cancer genetics specialists. Likewise, many health care provid- 
ers may have limited knowledge and experience in managing inherited cancer syndromes. ${ }^{10,11}$ These circumstances indicate a need to develop and evaluate cost-effective methods aimed at improving families' understanding, decision-making, and interpersonal communication about their risk for LFS.

Studies have evaluated the use of decision aids (DAs) as supplements to providers' counseling and as a means to facilitate informed choices for various cancer screening and treatment outcomes. ${ }^{12}$ DAs have been effective in increasing patients' knowledge and involvement in the decision-making process, although their impact on the actual choice of treatment options has varied. ${ }^{12}$ These findings are generally consistent with more recent studies that have evaluated DAs in the context of decision-making for BRCA1/BRCA2 genetic testing and subsequent risk management options. ${ }^{13-17}$ DAs may be particularly valuable adjuncts to cancer genetic counseling, as they may improve the efficiency of counseling sessions and enable counselors to focus more precisely on patients' individual risks, decisional preferences, and psychological concerns. ${ }^{18}$ Additional research on the use of such interventions specifically for cancer predisposition counseling and testing is needed.

To facilitate education and informed consent regarding clinical $p 53$ genetic counseling and testing among LFS-affected families, we developed and evaluated a video-based DA. The DA was intended to serve as a first-line intervention for LFS families who had previously participated in genetics research, and was offered as part of a longitudinal study of psychosocial and behavioral outcomes regarding $p 53$ genetic counseling and testing. The aim of the study reported here was to evaluate psychological outcomes and genetic testing decisions before and after use of the DA, and to determine the feasibility of delivering this type of intervention outside of a clinical setting. We hypothesized that after using the DA, participants would report: 1) improvements in knowledge, accuracy of perceived risk of carrying a $p 53$ mutation, and self-efficacy in regard to genetic counseling and testing; 2) no negative effects on mood; and 3 ) a reduction in decisional conflict regarding genetic testing.

\section{MATERIALS AND METHODS}

\section{Study population}

Our sample included 57 adults from 13 kindreds who had previously participated in research regarding the genetics of LFS at The University of Texas M. D. Anderson Cancer Center (MDACC) [Strong et al., unpublished data].4,19,20 The kindreds had initially been identified through systematic studies of childhood sarcoma patients, and a deleterious $p 53$ germline mutation had been identified in each kindred prior to the present study. Eligibility criteria included having donated a blood sample for research before clinical p53 mutation testing was available, having at least a $25 \%$ mutation carrier risk, being 18 years of age or older, and speaking English. Individuals with or without a personal history of cancer were eligible. In this study, "affected" persons included anyone with a prior diagnosis of any cancer excluding non-melanoma skin cancer, and "unaffecteds" included those with no personal history of cancer. No one had undergone genetic counseling or clinical p53 genetic testing prior to the present study.

\section{Study procedures}

This study was conducted as part of a protocol that offered genetic counseling and clinical $p 53$ genetic testing, and was approved by the MDACC Institutional Review Board. One hundred and five eligible persons were mailed a letter of invitation to participate in a psychosocial study and to receive genetic counseling about their personal and familial cancer risk, with the option of genetic testing. Study staff followed up with potential participants by telephone to determine their interest in participating. Seventy-three gave informed consent to participate in the psychosocial study and completed a baseline questionnaire by telephone; the remaining 32 declined, did not return follow-up telephone calls, or could not be reached. After completing the baseline questionnaire, participants were offered a video-based DA on hereditary cancer and genetic testing, with a specific focus on LFS, and were asked to participate in a post-DA telephone assessment. Sixty-eight agreed to receive the DA and were mailed a copy immediately after the completion of the baseline interview. Study staff followed up with participants by telephone within approximately two weeks after mailing the DA to determine whether participants had received and viewed the video, and to administer the post-DA questionnaire. Study staff encouraged participants who had not yet viewed the video-based DA to do so, and followed up with those participants at a mutually agreed-upon time to complete the post-DA interview. The average length of time between completion of baseline and post-DA assessments was six weeks. Fifty-seven completed the post-DA questionnaire and comprised our final study sample. Of the eleven individuals who agreed to receive the DA but who did not complete the post-DA assessment, three declined to continue in the study and eight did not view the DA or were unavailable to complete the follow-up interview.

After completion of the post-DA interview, study staff informed participants of options for receiving genetic counseling, and recorded their decisions. Our study population was geographically dispersed, and we offered persons the opportunity to either receive genetic counseling at MDACC at no cost, or to be referred to a genetic counselor closer to their home with the possibility that they might incur a cost for this service. All p53 genetic testing was performed at no cost. Uptake of genetic testing was recorded for those participants who completed pretest genetic counseling. Individuals who did not participate in the questionnaire or DA components of the study also were offered genetic counseling and the option of $p 53$ genetic testing.

\section{Video-based decision aid}

The primary goals of the DA were to enhance individuals' understanding of the clinical and psychosocial aspects of LFS and to facilitate informed decision-making about clinical p53 genetic counseling and testing. The development of the DA 
was guided conceptually by models of decision-making, crisis theory, and genetic counseling, ${ }^{21-24}$ and comprised several steps, including: 1) a literature review on clinical and psychosocial aspects of inherited cancer syndromes and genetic counseling and testing, with a specific focus on LFS and $p 53$ mutation testing; 2) a review of existing resources for patients focusing on genetic counseling and testing decisions, including both prenatal and cancer predisposition counseling and testing; 3) a needs assessment involving persons who had previously undergone cancer genetic counseling and testing at MDACC; and 4) expert opinion from a multidisciplinary panel that included expertise in clinical genetics, oncology, genetic counseling, clinical ethics and behavioral science. Based on this preliminary work, we developed a 26 -minute video-based DA in English that included the following elements: information about LFS, options for cancer genetic testing, and the possible outcomes associated with the following topics: information about inherited cancers and LFS, including basic information about cancer biology and cancer genetics and principles of inheritance; genetic counseling and testing options for inherited cancers; a description of possible outcomes and the risks, benefits, limitations and implications of counseling and testing outcomes; and, psychosocial and ethical considerations regarding cancer genetic testing. In addition to the didactic presentation of information, the DA also included video segments of actual patients and their families discussing their experience with inherited cancer and genetic testing as examples of how others have considered counseling and testing decisions. These segments represented the range of possible testing decisions for both patients and their family members, opinions regarding those decisions, and issues that one might consider when deciding about counseling and testing.

\section{Measures}

Following informed consent, all measures were administered by telephone at baseline (prior to the DA and offer of genetic counseling), except items assessing satisfaction with the DA. All measures, except demographics and quality of life, were administered post-DA.

\section{Sociodemographic and medical characteristics}

We assessed age, gender, race/ethnicity, education, marital status, number of children, and geographic location. Personal and family cancer histories were obtained from study records.

\section{Psychological distress}

Validated, reliable measures of distress were used. The StateTrait Anxiety Inventory (STAI) is a 40-item scale, with two subscales that measure trait and state or transitory anxiety, respectively. ${ }^{25}$ Subscale scores range from 20 to 80, with higher scores indicating greater anxiety. The Center for Epidemiologic Studies Depression (CES-D) Scale 26,27 is a 20 -item measure that has been widely used in general population samples. Scores range from 0 to 60 , with higher scores indicating a greater frequency and/or intensity of depressive symptoms. A cut-off score of 16 or greater has been used to denote persons with depressive symptomatology.

\section{Cancer-specific distress}

The 15-item revised Impact of Event Scale (IES) measured distress specific to having a family history of cancer; namely, the extent to which a person is experiencing signs or symptoms of intrusive thoughts or periods of avoidance, blocking or denial of distress. ${ }^{28}$ Overall scores range from 0 to 75 , with higher scores indicating greater cancer-specific distress.

\section{Cancer worries}

We used a three-item scale that assesses current frequency of worry about developing cancer, and the impact of that worry on mood and the ability to perform daily activities. ${ }^{29}$ Scores range from 1 to 4 , with higher scores indicating more frequent worries about cancer.

\section{Perceived risk}

Perceived risk of developing cancer was assessed by a single item: "In your opinion, compared with other persons your age, would you say your chances of getting cancer are: $1=$ much lower, $2=$ a little lower, $3=$ about the same, $4=$ a little higher, $5=$ much higher." Persons with a prior personal history of cancer were asked to rate their perceived risk of developing cancer again. This item was adapted from a core set of measures recommended by the Measurement Task Force of the Cancer Genetics Studies Consortium. ${ }^{30}$ We also evaluated perceived risk of carrying a deleterious $p 53$ mutation on a continuous scale ranging from 0 to $100 \%$.

\section{Self-efficacy}

Self-efficacy, i.e., a person's self-confidence in his or her ability to undergo genetic counseling and testing, was measured using three items. Participants were asked to indicate, on a scale of 1 to $100 \%$, how confident they were that they could: undergo genetic counseling; complete genetic testing; and cope with the possibility that they were mutation carriers. Scores for each item ranged from 0 to 100 , with higher scores indicating greater self-efficacy.

\section{Quality of life}

Quality of life was measured using the Ferrans and Powers Quality of Life Index (QLI). The QLI is a multidimensional rating scale that measures satisfaction across four life domains, measured by family, health/functioning, psychological/spiritual, and socioeconomic subscales. Scores range from 0 to 30, and are calculated for the overall scale as well as each subscale.

\section{Decisional conflict}

We used the Decisional Conflict Scale ${ }^{31}$ to measure uncertainty or perceived difficulty in making a decision about genetic testing. Using a five-point Likert response format, this validated scale measures uncertainty in choosing among various decision alternatives, and factors that contribute to uncertainty such as feeling informed, feeling clear in one's values, 
and perceived support in decision-making. Perceived quality of decision-making also is measured. Items are summed and averaged to obtain scores ranging from 1 (low decisional conflict) to 5 (high decisional conflict).

\section{Knowledge}

Nineteen items, comprising true/false and multiple choice response formats, were developed by the investigator team to evaluate participants' knowledge of relevant topics in hereditary cancer and genetic testing, including: inherited predisposition to cancer; general cancer risks; benefits, risks and limitations of genetic testing; and screening for cancer. The knowledge items underwent review by an expert panel for appropriateness and clarity, and were pilot tested with a separate patient sample. The knowledge score reflected the mean percent of correct answers.

\section{Intention to undergo p53 genetic testing}

Intention was measured by one item that asked participants to indicate their plans for having genetic testing. Responses included: 1 = no thought of having genetic testing; 2 = think I need to consider having testing someday; $3=$ think I should have testing, but am not quite ready; $4=$ think I probably will have testing; $5=$ committed to having genetic testing. Scores ranged from 1 to 5 , with higher scores indicating greater intention to have testing.

\section{Satisfaction with DA}

Five multiple choice response items evaluated the amount of information, the length, the understandability, the balance and fairness, and the overall acceptability of the video-based DA. One item assessed the participants' willingness to recommend the DA to others.

\section{Analysis}

Participants who completed both the baseline and post-DA measures were included in all analyses $(n=57)$. Descriptive statistics (mean, median, and standard deviation) were computed for demographic characteristics and psychosocial measures. All analyses were performed with SAS PROC MIXED (SAS System for Mixed Models, SAS Institute Inc, 1996). Statistical significance was set at $P<0.05$.

\section{Baseline comparisons}

Univariate comparisons of demographic characteristics and baseline psychosocial measures were conducted between affected and unaffected participants, and these subgroups were combined for further analyses. Next, we evaluated correlates of intention to have genetic testing at baseline. The intention to test measure was dichotomized into committed to test/probably will test versus other (coded 0 or 1 ). Associations between intention to test and baseline variables were examined using two-sample $t$-tests for continuous variables and contingency tables with $\chi^{2}$ analyses for categorical variables. Fisher's exact tests were used as necessary. Variables that were associated with baseline intention at the $P<0.15$ level were evaluated further in a logistic regression model.

\section{Baseline and post-DA comparisons}

We evaluated differences between baseline and post-DA scores for all psychosocial variables except quality of life. The percentage of responses indicating 'committed/probably' on the intention to test measure, and mean scores on perceived cancer risk, knowledge, decisional conflict, cancer worry and depression were stratified by gender; baseline to post-DA comparisons were evaluated using repeated measures analysis of variance. To control for potential correlations among members of the same family, family of origin was entered as a random factor in the model. Intra-class correlation coefficients were calculated to determine the degree of correlation among members of the same family, and results indicated that any effects on the outcomes of interest due to family membership were minimal.

\section{RESULTS}

\section{Participant characteristics}

Most participants were Caucasian, and the majority were female, married, had completed at least some college, and had at least one biological child (Table 1). Seventy-five percent did not have a personal history of cancer. The majority resided outside of the Houston metropolitan area, and only $14 \%$ lived within 100 miles of MDACC. When compared with persons who declined the study and those who completed only the baseline interview, participants did not differ on age or race/ ethnicity. However, a significantly greater proportion of women completed both the baseline and post-DA assessments compared with men $(64 \%$ vs. $43 \%, P<0.05)$.

\section{Baseline comparisons and correlates of baseline intention to have genetic testing}

As shown in Table 2, affected and unaffected participants did not differ in terms of their baseline scores on the psychosocial measures, with two exceptions: affected participants

Table 1

Participants' demographic characteristics by cancer history status (affected vs. unaffected)

\begin{tabular}{lccc}
\hline Characteristic & $\begin{array}{c}\text { Affected } \\
(\mathrm{N}=14)\end{array}$ & $\begin{array}{c}\text { Unaffected } \\
(\mathrm{N}=43)\end{array}$ & $\begin{array}{c}\text { Total } \\
(\mathrm{N}=57)\end{array}$ \\
\hline $\begin{array}{l}\text { Mean age, } \\
\text { (SD, range), yrs }\end{array}$ & $\begin{array}{c}50.6 \\
(14.6,18-75)\end{array}$ & $\begin{array}{c}52.2 \\
(12.4,23-74)\end{array}$ & $\begin{array}{c}51.8 \\
(2.8,18-75)\end{array}$ \\
\cline { 2 - 4 } & $\mathrm{N}(\%)$ & $\mathrm{N}(\%)$ & $\mathrm{N}(\%)$ \\
Gender- Female & $11(79)$ & $25(58)$ & $36(63)$ \\
Race/ethnicity- White & $13(93)$ & $36(84)$ & $49(86)$ \\
Education $\geq$ high school & $8(57)$ & $29(67)$ & $37(65)$ \\
Married & $8(62)$ & $29(67)$ & $37(65)$ \\
$>1$ biological child & $10(71)$ & $38(93)$ & $48(87)$ \\
\hline
\end{tabular}


Table 2

Participants' baseline scores (M, SD) on psychosocial measures by personal cancer history status (affected vs. unaffected), $\mathrm{N}=57$

\begin{tabular}{lcc}
\hline Measure, Range & $\begin{array}{c}\text { Unaffected } \\
(\mathrm{N}=43)\end{array}$ & $\begin{array}{c}\text { Affected } \\
(\mathrm{N}=14)\end{array}$ \\
\hline Anxiety (STAI) & & \\
State, 20-80 & $27.2(7.3)$ & $24.0(4.6)$ \\
Trait, 20-80 & $32.9(9.5)$ & $30.0(6.4)$ \\
Depression (CES-D), 0-60 & $8.1(7.5)$ & $6.6(7.8)$ \\
Cancer-specific distress (IES), 0-75 & $10.0(13.0)$ & $8.4(9.7)$ \\
Cancer worries, 1-4 & $1.3(0.4)$ & $1.6(0.7)$ \\
Perceived risk & & \\
Developing cancer, 1-5 ${ }^{a}$ & $3.4(1.1)$ & $4.2(1.3)$ \\
Having a P53 mutation, 0-100\% ${ }^{a}$ & $41.8(23.7)$ & $61.4(29.7)$ \\
Self-efficacy, 0-100\% & & \\
Have genetic counseling & $85.2(18.2)$ & $92.1(14.7)$ \\
Have genetic testing & $85.9(18.2)$ & $89.9(21.8)$ \\
Cope with positive test result & $84.4(20.9)$ & $90.9(16.3)$ \\
Quality of life, 0-30 & $22.4(3.9)$ & $22.9(4.1)$ \\
Decisional conflict, 1-5 & $2.2(0.7)$ & $2.1(0.6)$ \\
Knowledge, 0-100\% correct & $67 \%$ & $65 \%$ \\
Intention to test - \% & $74 \%$ & $79 \%$ \\
$\quad$ committed/probably & & \\
\hline${ }^{a}$ < 0.05 & &
\end{tabular}

${ }^{a} P<0.05$.

scored significantly higher on measures of perceived risk of developing cancer and carrying a $p 53$ germline mutation. For all participants, mean scores on measures of both general and cancer-specific distress were generally within normal ranges. Twelve percent had scores of 16 or higher on the CES-D. Mean knowledge scores indicated that participants may lack information on some aspects of LFS or genetic testing, and over three-fourths indicated an intention to have genetic testing. To evaluate correlates of baseline testing intention, variables that were associated with intention at the $P<0.15$ level in univariate analysis were entered into a multivariate regression model (Table 3). Decisional conflict was the only variable associated with greater baseline intention to have testing; those indicating

Table 3

Results of logistic regression analysis evaluating correlates of baseline intention to have p53 genetic testing, $\mathrm{N}=57$

\begin{tabular}{lccc}
\hline Variable & OR & $95 \%$ CI & $p$ \\
\hline $\begin{array}{l}\text { Decisional conflict } \\
\text { Perceived risk }\end{array}$ & 0.17 & $(0.04,0.65)$ & 0.010 \\
$\quad$ Having a $p 53$ mutation & 1.01 & $(0.98,1.04)$ & 0.462 \\
Self efficacy & & & \\
$\quad$ Have genetic counseling & 1.01 & $(0.97,1.05)$ & 0.667 \\
Gender- Female & 3.1 & $(0.73,13.23)$ & 0.126 \\
\hline
\end{tabular}

lower decisional conflict were 5.9 times more likely to express a stronger intention to have testing compared with those indicating greater decisional conflict. The total variance explained by the model was $34 \%$.

\section{Psychosocial and decision outcomes}

As shown in Table 4, knowledge scores increased significantly for both men and women $(P<0.01)$ from baseline to post-DA assessment, and mean scores on measures of decisional conflict, perceived risk of developing cancer, and cancer worries decreased significantly $(P<0.01)$. Also among men, mean scores on the CES-D decreased significantly from baseline to post-DA, and men's post-DA mean depression scores were significantly lower compared with women's scores. Participants' intention to have genetic testing did not change following the use of the DA.

Thirty-nine (68\%) participants underwent pre-test genetic counseling, and 23 (40\%) donated a blood sample for clinical testing with the intention of receiving test results. In a separate analysis using two-sample $t$-tests, we found that participants who underwent genetic counseling had lower decisional conflict scores and indicated greater testing intention compared with those who did not follow through with counseling $(P<$ 0.05 ). Participants who gave a blood sample for clinical testing were more likely to be female, and had lower decisional conflict scores as well as higher mean scores on cancer worries, perceived risk of cancer, and intention to test compared with those who had not followed through with counseling or testing $(P<$ $0.001)$.

\section{Satisfaction with DA}

Seventy-seven percent or more reported that the length of the DA and the amount of information presented was appropriate, and that the content was easy to comprehend. Most (70\%) agreed that the DA presented the option of genetic testing in a balanced way, while others indicated it was slightly $(21 \%)$ or very $(7 \%)$ biased to testing. Ninety-six percent reported that they would recommend the DA to others who were considering genetic counseling and testing.

\section{DISCUSSION}

This study demonstrated that a video-based DA offered prior to genetic counseling and testing for LFS is feasible and well-accepted, and that it may have a favorable effect on several psychological factors that may be important in both decisionmaking about counseling and testing as well as in coping with outcomes of those processes. Our findings support the use of this method as a first-line intervention for LFS families who may be considering genetic counseling and testing. We demonstrated that the DA improved knowledge about hereditary cancer and genetic testing among persons from LFS families, which is consistent with earlier research. ${ }^{12}$ Our results also suggest that delivering this information through a self-administered method outside of a clinic setting is feasible and may not have a negative effect on mood or cancer-related distress and 
Table 4

Baseline vs. post-decision aid (DA) comparison of participants' scores on psychosocial measures by gender, $\mathrm{N}=57$

\begin{tabular}{|c|c|c|c|c|}
\hline \multirow[b]{2}{*}{ Measure, Range } & \multicolumn{2}{|c|}{ Males $(\mathrm{N}=21)$} & \multicolumn{2}{|c|}{ Females $(\mathrm{N}=36)$} \\
\hline & Baseline & Post-DA & Baseline & Post-DA \\
\hline \multirow[t]{2}{*}{ Intention to test, $\%$ committed/probably } & $62 \%$ & $67 \%$ & $83 \%$ & $83 \%$ \\
\hline & $\mathrm{M}(\mathrm{SD})$ & $\mathrm{M}(\mathrm{SD})$ & $\mathrm{M}(\mathrm{SD})$ & $\mathrm{M}(\mathrm{SD})$ \\
\hline State anxiety, $20-80$ & $26.9(8.3)$ & $25.5(5.7)$ & $26.2(6.0)$ & $28.0(8.0)$ \\
\hline Depression (CES-D), $0-60^{a}$ & $7.4(8.1)$ & $3.2(4.1)$ & $7.9(7.3)$ & $8.6(9.1)$ \\
\hline Cancer-specific distress (IES), 0-75 & $8.9(12.8)$ & $6.2(9.6)$ & $10.0(12.1)$ & $10.6(13.8)$ \\
\hline Cancer worries, $1-4^{b}$ & $1.3(0.5)$ & $1.0(0.4)$ & $1.4(0.5)$ & $1.0(0.5)$ \\
\hline \multicolumn{5}{|l|}{ Perceived risk } \\
\hline Developing cancer, $1-5^{b}$ & $3.3(1.2)$ & $2.4(1.2)$ & $3.7(1.1)$ & $2.5(1.0)$ \\
\hline Carrying a $p 53$ mutation, $0-100 \%$ & $41.0(28.2)$ & $39.9(24.6)$ & $48.5(24.1)$ & $55.4(30.0)$ \\
\hline \multicolumn{5}{|l|}{ Self-efficacy, 0-100\% } \\
\hline Have genetic counseling & $83.3(18.5)$ & $81.0(30.8)$ & $88.8(17.3)$ & $91.1(18.0)$ \\
\hline Have genetic testing & $82.1(27.4)$ & $92.8(21.9)$ & $88.7(19.8)$ & $90.6(21.7)$ \\
\hline Cope with positive test result & $85.2(23.5)$ & $81.9(34.8)$ & $85.3(18.3)$ & $85.3(21.7)$ \\
\hline Decisional conflict, $1-5^{b}$ & $2.3(0.5)$ & $1.8(0.6)$ & $2.1(0.7)$ & $1.8(0.6)$ \\
\hline Knowledge, $0-100 \%$ correct $^{b}$ & $67 \%$ & $77 \%$ & $66 \%$ & $75 \%$ \\
\hline
\end{tabular}

${ }^{a} P<0.05$ (baseline to post DA), males only.

${ }^{b} P<0.01$ (baseline to post DA), males and females.

worries. We observed decreases in cancer worries scores for all participants following use of the DA. Mean depression scores also decreased among men; however, it is important to note that both baseline and post-DA scores were below the cutoff for consideration of clinical referral. Nonetheless, these findings suggest that initial education prior to genetic counseling may have psychological benefits, perhaps particularly for those who had previously participated in genetics research and are contemplating clinical genetic counseling and testing. Interventions such as the video-based DA can address critical knowledge deficits and also may show some benefit in reducing distress, which may be barriers to seeking genetic counseling and testing ${ }^{32}$ as well as to comprehension of risk information and adherence to surveillance recommendations.

Although use of the DA improved knowledge, we observed no change in participants' intentions to have genetic counseling or testing. Participants reported lower levels of decisional conflict post-DA, which suggests that the DA may have helped reduce uncertainty and improve the perceived effectiveness of genetic counseling and testing decisions. The percentage of persons who followed through with genetic counseling and donation of a blood sample for clinical testing was lower than the percentage of persons who indicated an intention to do so at the post-DA assessment. Studies have shown that expressed intention to undergo predictive genetic testing may not consistently predict actual uptake. ${ }^{33}$ However, other research suggests that intention and actual uptake of testing may be more closely aligned among persons at highest risk of carrying a cancer-predisposing mutation, ${ }^{14}$ such as our study population, all of whom were at high risk of carrying a $p 53$ mutation. It is important to note that while we have evaluated counseling and testing decisions at a given point in time as part of this study, some individuals may in fact make these decisions over a longer interval. Additional external factors such as family context variables and other relatives' experiences with genetic testing also may influence individual decisions regarding counseling and testing. ${ }^{34}$ As part of our ongoing longitudinal study, we will continue to monitor participants over time to determine their preferences and choices regarding p53 counseling and testing.

The genetic counseling and testing process involves multiple decisions, from the initial choice to seek information about one's hereditary cancer risk to the decision to receive one's test results and eventually follow through with risk management recommendations. The initial decision to obtain information about one's personal risk for inherited cancer may in fact be motivated by the desire to obtain this information for the benefit of other family members. ${ }^{33}$ While patients generally express a willingness to share genetic risk information with their family members, perceived difficulty in conveying such information and a lack of emotional closeness with family members may pose barriers to family communication. ${ }^{35,36}$ An advantage of the DA developed for this study is that it may be an easy, non-threatening way to share information about LFS and genetic testing with family members to facilitate informed decision-making.

Our findings add evidence to the existing literature regarding the potential usefulness of DAs in cancer genetic counsel- 
ing and testing. ${ }^{14,16,17}$ The need for resources to educate persons and inform decision-making about hereditary cancers and genetic testing may be especially important for LFS families, given the rare nature of this syndrome, the broad spectrum of tumors and cancer risks in LFS, and the potential complexity of surveillance regimens. Methods that enhance individuals' understanding and awareness of LFS can be an important and essential first step to identifying affected families who may benefit from genetic counseling and risk assessment services. For example, a recent study indicated that, with the exception of breast cancer, LFS-associated cancers tend to be inaccurately reported or underreported by families with $p 53$ mutations compared to families with hereditary breast and ovarian cancer syndrome. ${ }^{37}$ In that study, only $42 \%$ of historians from LFS families provided cancer family histories that met the criteria for LFS; based on the information provided, a substantial number of families may not have been referred for a genetics evaluation. Genetic risk information may not be conveyed accurately within families, and such communication may be limited largely to the nuclear, or immediate, family. ${ }^{35,38}$ Resources such as our DA have the potential to help facilitate awareness and accurate communication about hereditary cancer risks within LFS families.

The video-based format was an appropriate method for reaching members of LFS families in this study. Although more interactive formats, such as Web-based or CD-ROM-based decision aids, may seem more engaging and can be tailored to individual's informational needs, a video-based format may be more accessible to a greater number of people. Although the families included in the video-based DA represented a range of experiences and decisions regarding genetic testing, we were not able to include an ethnically diverse sample of families; thus, it may be somewhat limited in its appeal to broader audiences. Future research should attempt to adapt the DA for more diverse audiences, to evaluate it in emerging technologies, and to enhance the delivery with additional self-guided materials that further help clarify and incorporate the consideration of values in determining decision preferences. ${ }^{39-41}$

The results of this study are encouraging; however, limitations must be noted. The study employed a one-arm, nonrandomized design. Randomizing participants to DA versus control conditions at either the individual or family level was not feasible, given the small number of affected families in this study as well as the inability to control for possible contamination within families. Our sample included a relatively small number of families, all of whom had previously participated in research on the genetics of LFS, which may limit the generalizability of our findings. Future research should involve further evaluation of such DAs in randomized controlled trials with larger, diverse samples. Nonetheless, our findings are important because they demonstrate the feasibility and usefulness of offering this type of intervention in a non-clinic setting, which may help in reaching members of hereditary cancer families who are geographically dispersed and who reside in areas that may be underserved in terms of cancer genetics services. Given the increasing role of genomics in public health and medical care, outreach efforts using interventions such as ours will be important in assuring that persons have the knowledge to make initial decisions about genetic counseling and testing options for themselves and their families.

\section{ACKNOWLEDGMENTS}

Supported by the American Cancer Society MRSGT-04204-01-CPHHPS (Peterson), the National Cancer Institute, National Institutes of Health CA34936 (Strong), and the Kleberg Human Cancer Genetics Development Award (Pentz and Strong). We thank Sapna Kapoor, MiWon Park, Jenny Patel, Gloria Robertson, and Doris Sembera, who assisted with data collection. Finally, we thank the participants for their valuable contributions to our study.

\section{References}

1. Frebourg T, Barbier N, Yan YX, Garber JE, et al. Germ-line p53 mutations in 15 families with Li-Fraumeni syndrome. Am J Hum Genet 1995;56:608-615.

2. Varley JM, McGown G, Thorncroft M, Santibanez-Koref MF, et al. Germ-line mutations of TP53 in Li-Fraumeni families: An extended study of 39 families. Cancer Research 1997;57:3245-3252.

3. Bougeard G, Limacher JM, Martin C, Charbonnier F, et al. Detection of 11 germline inactivating TP53 mutations and absence of TP63 and HCHK2 mutations in 17 French families. J Med Genet 2001;38:253-257.

4. Hwang SJ, Lorano G, Amos CI, Strong LC. Germline p53 mutations in a cohort with childhood sarcoma: Sex differences in cancer risk. Am J Hum Genet 2003;72:975983.

5. Chompret A, Brugieres L, Ronsin M, Gardes M, et al. P53 germline mutations in childhood cancers and cancer risk for carrier individuals. British Journal of Cancer 2000;82:1932-1937.

6. Schneider KA, Li F. Li-Fraumeni syndrome. Gene Reviews, 2004.

7. Varley JM, Evans DGR, and Birch JM. Li-Fraumeni syndrome: A molecular and clinical review. British Journal of Cancer 1997;76:1-14.

8. Patenaude AF, Schneider KA, Kieffer SA, Calzone KA, et al. Acceptance of invitations for $\mathrm{p} 53$ and BRCA1 predisposition testing: Factors influencing potential utilization of cancer genetic testing. Psycho-oncology 1996;5:241-250.

9. Dorval M, Patenaude AF, Schneider KA, Kieffer SA, et al. Anticipated versus actual emotional reactions to disclosure of results of genetic tests for cancer susceptibility: Findings from p53 and BRCA1 testing programs. Journal of Clinical Oncology 2000; 18:2135-3142.

10. NSGC, National Society of Genetic Counselors Familial Cancer Risk Counseling Special Interest Group Directory. 2003, Wallingford, PA: National Society of Genetic Counselors.

11. Freedman AN, Wideroff L, Olson L, Davis A, et al. U.S. physicians' attitudes toward genetic testing for cancer susceptibility. Am J Med Genet, 2003;120:63-71.

12. O'Connor A, Stacey D, Entwistle V, Llewellyn-Thomas H, et al. Decision aids for people facing health treatment or screening decisions. Cochrane Database Syst Rev 2003; (2):CD001431. Review.

13. Green MJ, McInerney AM, Biesecker BB, and Fost N. Education about genetic testing for breast cancer susceptibility: Patient preferences for a computer program or genetic counselor. Am J Med Genet 2001;103:24-31.

14. Green MJ, Peterson SK, Baker MW, Harper GR, et al. Effect of a computer-based decision aid on knowledge, perceptions, and intentions about genetic testing for breast cancer susceptibility: a randomized controlled trial. Journal of the American Medical Association 2004;292:442-452.

15. van Roosmalen MS, Stalmeier PF, Verhoef LC, Hoekstra-Weebers JE, et al. Randomised trial of a decision aid and its timing for women being tested for a BRCA1/2 mutation. Br J Cancer 2004;90:333-342.

16. Schwartz MD, Benkendorf J, Lerman C, Isaacs C, et al. Impact of educational print materials on knowledge, attitudes, and interest in BRCA1/BRCA2: testing among Ashkenazi Jewish women. Cancer 2001;92:932-940.

17. Lerman C, Biesecker B, Benkendorf JL, Knrner J, et al. Controlled trial of pretest education approaches to enhance informed decision-making for BRCA1 gene testing. J Natl Cancer Inst 1997;89:148-157.

18. Green MJ, Peterson SK, Baker MW, Friedman LC, et al. Use of an educational computer program before genetic counseling for breast cancer susceptibility: effects on duration and content of counseling sessions. Genet Med 2005;7:221-229. 
19. Strong LC, Stine M, and Norsted TL. Cancer in survivors of childhood soft tissue sarcoma and their relatives. J Natl Cancer Inst 1987;79:1213-1220.

20. Lustbader ED, Williams WR, Bondy ML, Strom S, et al. Segregation analysis of cancer in families of childhood soft-tissue sarcoma patients. Am J Hum Genet 1992; 51:344-356.

21. Kessler S. Genetic counseling: Psychological dimensions. 1979, New York: Academic Press.

22. Janis IL, Mann L. Decision Making: A psychological analysis of conflict, choice, and committment, N.Y.F. Press, Editor: New York, 1977.

23. Ajzen I, Fishbein M. Understanding attitudes and predicting social behavior. 1980, Englewood Cliffs, NJ: Prentice Hall.

24. Moos RH, Schaefer JA. The crisis of physical illness. An overview and conceptual approach. In Moos, R.H. (Ed). Coping with physical illness. Two new perspectives. Plenum Medical Book Co: New York; 1984, 3-25.

25. Spielberger CD, Gorsuch RL, Lushene RE, Vagg PR, et al. State-Trait Anxiety Inventory Manual. 1970, Palo Alto, CA: Counsulting Psychologists Press.

26. Radloff LS. The CES-D scale: A self-report depression scale for research in the general population. Applied Psychological Measurement 1977;1:385-401.

27. Weissman MM, Sholomskas D, Pottenger M, Prusoff BA, et al. Assessing depressive symptoms in five psychiatric populations: a validation study. Am J Epidemiol 1977; 106:203-214.

28. Horowitz M, Wilner N, and Alvarez W. Impact of event scale: A measure of subjective stress. Psychosomatic Medicine 1979;41:209-218.

29. Lerman C, Trock B, Rimer BK, Boyce A, et al. Psychological and behavioral implications of abnormal mammograms. Ann Intern Med 1991;114:657-661.

30. Bowen DJ, Patenaude AF, and Vernon SW. Psychosocial issues in cancer genetics: from the laboratory to the public. Cancer Epidemiology, Biomarkers and Prevention $1999 ; 8: 326-328$
31. O'Connor AM. Validation of a decisional conflict scale. Medical Decision Making 1995; 15:25-30.

32. Lerman C, Hughes C, Trock BJ, Myers RE, et al. Genetic testing in families with hereditary nonpolyposis colon cancer. Journal of the American Medical Association 1999;281:1618-1622.

33. Lerman C, Croyle RT, Tercyak KP, and Hamann H. Genetic testing: psychological aspects and implications. Journal of Consulting and Clinical Psychology 2002;70:784-797.

34. Peterson SK. The role of the family in genetic testing: Theoretical perspectives, current knowledge, and future directions. Health Education \& Behavior 2005;32: $627-639$.

35. Wilson BJ, Forrest K, van Teijlingen ER, McKee L, et al. Family Communication about Genetic Risk: The Little That Is Known. Community Genetics 2004;7:15-24.

36. Gaff CL, Collins V, Symes T, Halliday J. Facilitating family communication about predictive genetic testing: Probands' perceptions. Journal of Genetic Counseling 2005;14:133-140.

37. Schneider KA, DiGianni LM, Patenaude AF, Klar N, et al. Accuracy of cancer family histories: comparison of two breast cancer syndromes. Genetic Testing 2004;8:222228.

38. Peterson SK, Watts BG, Koehly LM, Vernon SW, et al. How families communicate about HNPCC genetic testing: Findings from a qualitative study. Am J Med Genet 2003;119:78-86.

39. Tiller K, Meiser B, Reeson E, Tucker M, et al. A decision aid for women at increased risk for ovarian cancer. Int J Gynecol Cancer 2003;13:15-22.

40. Charles C, Gafni A, Whelan T, O'Brien MA. Treatment decision aids: conceptual issues and future directions. Health Expect 2005;8:114-25.

41. O'Cathain A, and Thomas KJ. Evaluating decision aids-where next? Health Expectations 2004;7:98-103. 